\title{
Water Situation is Known
}

\author{
Zheng $\mathrm{Hu}$ \\ No. 689, Huadian Road, Beishi District, Baoding City, Hebei Province, China \\ 1415037523@qq.com
}

Keywords: water scarcity, monadic regression, multiple linear regression, water supply ability

Abstract .Water Supply has drawn a great deal of attention from the entire world for recent decades, while the available water is scarce in many countries and regions.

The aim is to build a mathematical model to measure the ability of a region to provide clean water to meet the needs of its population, and use the model to make a prediction of the water situation for China's Jiangsu Province in 15 years.

Therefore, water demand and supply, were researched. As for water demand (have four influence factors: population density, factory distribution, industrial water consumption and agricultural water consumption), I set up monadic regression equations about the time variables for each factor. Then these four equations were added together to get a formula about time and water demand. For water supply (have four influence factors: sewage quantity, surface water, underground water and precipitation), according to the natural elements and environmental purification ability as well as the situation of the pollution of clean water getting from the severe polluted water, I found relevant data of all time variables. Based on the data, I set up a multiple linear regression equation about time variable for fresh water supply. Finally, on the basis of demand and supply, the water supply ability was defined as the measuring index.

\section{Introduction}

Clean, fresh water is the security for development in countries. According to the criterion of the International Per capita water resources, Jiangsu Province is a region with serious water scarcity that the water resources per capita is less than 500 cubic meters. Water resources utilization in Jiangsu Province is not high, industrial water consumption is higher than the north about 10 cubic meters in every 10,000 Yuan output value. The amount of water deficit is 2.46 billion cubic meters during the generally arid years, up to 10.27 billion cubic meters during the special arid years. Distribution of water resources in Jiangsu is not balanced and can't match with population, land and economic layout.

Meanwhile, water pollution of Jiangsu is also the most serious in China. The sewage discharge at unit area greatly surpasses nation's mean value, over 70\% sections exceed II kinds of water standards. Water pollution has become a major constraint to the achievement of sustainable socio-economic development. [1] 
Faced with water security and pollution, it is essential to build perfect water strategy to first know and predict the water situation timely.

\section{Process Analysis}

I analyze the factors affecting the water supply and demand, use the multivariate linear regression to study the impact of these factors on the water supply and demand, and then in a linear fitting method to determine the relationship between all kinds of factors and population.

Connection between population and year

$$
p=\beta_{1}\left(t-t_{0}\right)+\beta_{2}
$$

- Connection between population and some factors

Easy to use $\boldsymbol{x}_{\mathbf{1}}$ to $\boldsymbol{x}_{\mathbf{7}}$ express different factors. Among them, $\boldsymbol{x}_{\mathbf{1}}$ to $\boldsymbol{x}_{\mathbf{4}}$ influence the water supply, and $x_{\mathbf{5}}$ to $\boldsymbol{x}_{\mathbf{7}}$ influence the water demand, I get the following relationships through linear fitting:

$$
\left\{\begin{array}{l}
x_{1}=\mathrm{k}_{1} p+\mathrm{b}_{1} \\
x_{2}=\mathrm{k}_{2} p+\mathrm{b}_{2} \\
x_{3}=\mathrm{k}_{3} p+\mathrm{b}_{3} \\
x_{4}=\mathrm{k}_{4} p+\mathrm{b}_{4} \\
x_{5}=\mathrm{k}_{5} p+\mathrm{b}_{5} \\
x_{6}=\mathrm{k}_{6} p+\mathrm{b}_{6} \\
x_{7}=\mathrm{k}_{7} p+\mathrm{b}_{7}
\end{array}\right.
$$

- Different factors influence the supply and demand of water

Two formulas are as follows:

$$
\left\{\begin{array}{l}
y_{1}=\sum_{i=1}^{4} \alpha_{i} x_{i}+\varepsilon_{1} \\
y_{2}=\sum_{i=5}^{7} \alpha_{i} x_{i}+\varepsilon_{2}
\end{array}\right.
$$

Based on Equation 2, I can get:

$$
\left\{\begin{array}{l}
y_{1}=\left(\sum_{i=1}^{4} \alpha_{i} k_{i}\right) p+\sum_{i=1}^{4} \alpha_{i} b_{i}+\varepsilon_{1} \\
y_{2}=\left(\sum_{i=5}^{7} \alpha_{i} k_{i}\right) p+\sum_{i=5}^{7} \alpha_{i} b_{i}+\varepsilon_{2}
\end{array}\right.
$$

- Connection between the ability and the supply and demand

I want to build a relationship between supply and demand, with the difference between the supply and demand and the ratio of the difference and water supply to show ability, there are:

$$
\eta=y_{1}-y_{2} / y_{1}
$$

- Connection between the ability and the population

$$
\eta=\frac{\left(\sum_{i=1}^{4} \alpha_{i} k_{i}-\sum_{i=5}^{7} \alpha_{i} k_{i}\right) p+\left(\sum_{i=1}^{4} \alpha_{i} b_{i}-\sum_{i=5}^{7} \alpha_{i} b_{i}\right)+\left(\varepsilon_{1}-\varepsilon_{2}\right)}{\left(\sum_{i=1}^{4} \alpha_{i} k_{i}\right) p+\sum_{i=1}^{4} \alpha_{i} b_{i}+\varepsilon_{1}}
$$

- Final result 


$$
\eta=\frac{\left(\sum_{i=1}^{4} \alpha_{i} k_{i}-\sum_{i=5}^{7} \alpha_{i} k_{i}\right)\left[\beta_{1}\left(t-t_{0}\right)+\beta_{2}\right]+\left(\sum_{i=1}^{4} \alpha_{i} b_{i}-\sum_{i=5}^{7} \alpha_{i} b_{i}\right)+\left(\varepsilon_{1}-\varepsilon_{2}\right)}{\left(\sum_{i=1}^{4} \alpha_{i} k_{i}\right)\left[\beta_{1}\left(t-t_{0}\right)+\beta_{2}\right]+\sum_{i=1}^{4} \alpha_{i} b_{i}+\varepsilon_{1}}
$$

\section{Solving, testing, and predicting}

\subsection{Solving and testing(Take Jiangsu, China as an example)}

By searching for information, I find a large amount of data in statistical yearbook of Jiangsu 2000-2015

\section{- Connection between population and year}

Through fitting, I obtained that $\beta_{1}=0.4944$ and $\beta_{2}=73.3390$. According to the data, $t_{0}=2000$. Now, I can rewrite Equation 1 to

$$
p=0.4944(t-2000)+73.3390
$$

The fitting is good.

\section{- Connection between population and some factors}

A simple model was chosen by us to fit population and seven factors below.

$$
\left\{\begin{array}{rr}
x_{1}= & 0.0023 p+76.1093 \\
x_{2}= & -0.0536 p+82.9314 \\
x_{3}= & 0.1803 p+68.7045 \\
x_{4}= & -0.0018 p+78.6175 \\
x_{5}= & 0.9636 p+64.4103 \\
x_{6}= & 1.3232 p+50.0325 \\
x_{7}= & 1.1794 p+73.2788
\end{array}\right.
$$

\section{- Different factors influence the supply and demand of water}

Using multiple linear regression analysis to solve, I get the result:

$$
\begin{gathered}
y_{1}=-50.0865+0.9540 x_{1}+0.9786 x_{2}+0.2041 x_{3}+0.0375 x_{4} \\
y_{2}=404.8352-4.3830 x_{1}+2.1704 x_{2}+29.0697 x_{3}
\end{gathered}
$$

Through analysis $y_{1}$ is available, while $y_{2}$ is essential to optimize. Delete the 4 th number and analyze the data again, I get

$$
y_{2}=351.5574-20.6179 x_{1}+9.9751 x_{2}+27.5217 x_{3}
$$

Delete the 4th number again and still analyze the data, I get

$$
y_{2}=-4.7901+0.7344 x_{1}+1.5667 x_{2}+0.2892 x_{3}
$$

I also use correlation index to analyze error, such index of $y_{1}$ is equal to 0.7815 , and index of $y_{2}$ is equal to 0.8798.I can infer that those two equations are acceptable.

\section{- Final result}

Easy to find $\eta<0, \eta$ is to show ability, so that formulas can be changed to

$$
\eta=\frac{3.13533 p+21.81352}{-0.01353 p+120.2774}
$$

\subsection{Prediction}


Table 1. Connection among $p, t$ and $\eta$

\begin{tabular}{|c|c|c|c|c|c|c|c|}
\hline $\boldsymbol{\eta}_{\boldsymbol{p}}$ & 2.1070 & 2.1153 & 2.1277 & 2.1415 & 2.1588 & 2.1760 & 2.1939 \\
\hline $\boldsymbol{\eta}_{\boldsymbol{t}}$ & 52.7985 & 52.8170 & 52.8354 & 52.8539 & 52.8723 & 52.8908 & 52.9092 \\
\hline $\boldsymbol{\eta}_{\boldsymbol{p}}$ & 0.2364 & 0.2366 & 0.2369 & 0.2373 & 0.2377 & 0.2382 & 0.2386 \\
\hline $\boldsymbol{\eta}_{\boldsymbol{t}}$ & 22.1757 & 22.1759 & 22.1762 & 22.1764 & 22.1767 & 22.1769 & 22.1772 \\
\hline
\end{tabular}

Seen from the Table 1 I can know the water supply capacity of Jiangsu is not optimistic .I obtain the relationship between $x_{i}$ and $t$ from Equation 8 and Equation 9 .

$$
\left\{\begin{array}{rr}
x_{1}= & 0.0011 t+74.0039 \\
x_{2}= & -0.0265 t+131.9971 \\
x_{3}= & 0.0891 t-96.3429 \\
x_{4}= & -0.0009 t+70.3522 \\
x_{5}= & 0.4764 t-817.6736 \\
x_{6}= & 0.6512 t-1161.2309 \\
x_{7}= & 0.5831 t-1006.3494
\end{array}\right.
$$

Through analysis I bring $t=2031$ to Equation 15 and get different $x$ in Figure 1.

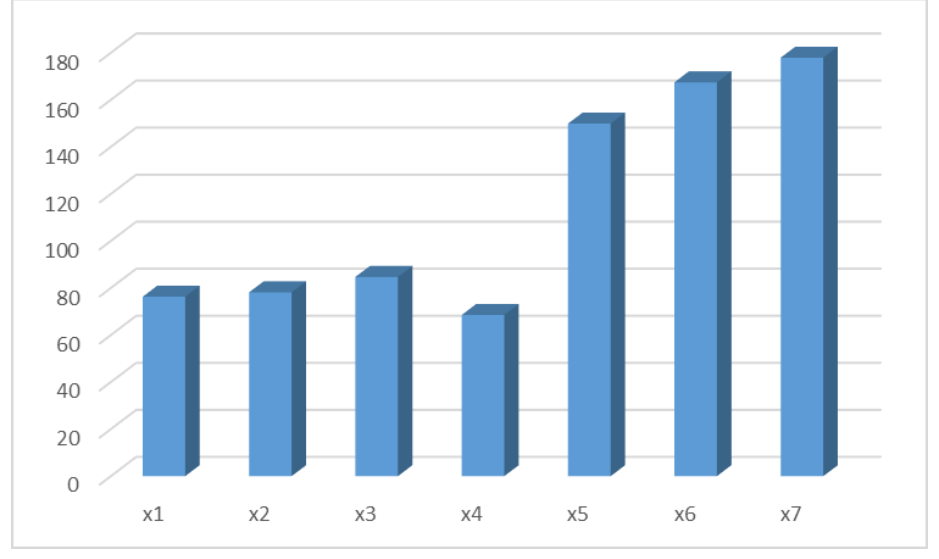

Figure 1. Different $x$

Based on Equation 15, Figure 1 and all former analysis, I conclude that Jiangsu's ability to provide water will fell slightly in 15 years. However, I think 15 years may be a turning point, after that, the situation may be better.

\section{References}

[1]

Using the

$\mathrm{UN}$

water

scarcity

map

http://www.unep.org/dewa/vitalwater/jpg/0222waterstress-overuse-EN.jpg

[2] Local Chronicles of Jiangsu province

http://www.jssdfz.gov.cn/index.php?m=content\&c=index\&a=show\&catid=9\&id=27

[3] Zhang Jiatao, The problems and countermeasures of sustainable utilization of water resources in Jiangsu. Jiangsu water resource,2002.N0.7

[4] Du Guiyang, Jiangsu urbanization in water conservation, The 2004 Beijing international academic conference on urban water conservancy construction and development, Page 338 